\title{
Hematopoietic Progenitor Cells from Apheresis
}

National Cancer Institute

\section{Source}

National Cancer Institute. Hematopoietic Progenitor Cells from Apheresis. NCI

Thesaurus. Code C133328.

Peripheral blood collected by apheresis as a source of hematopoietic progenitor cells. 